\title{
Textile Diamond Dipole and Artificial Magnetic Conductor Performance under Bending, Wetness and Specific Absorption Rate Measurements
}

\author{
Kamilia KAMARDIN ${ }^{1}$, Mohamad Kamal A. RAHIM ${ }^{2}$, Peter S. HALL ${ }^{3}$, \\ Noor Asmawati SAMSURI ${ }^{2}$, Mohd Ezwan JALIL ${ }^{2}$, Mohd Fareq ABD MALEK ${ }^{4}$ \\ ${ }^{1}$ Computer Systems Engineering Group, Advanced Informatics School, Universiti Teknologi Malaysia, \\ 54100 UTM KL, Kuala Lumpur, Malaysia \\ ${ }^{2}$ Dept. of Communication Engineering, Faculty of Electrical Engineering, Universiti Teknologi Malaysia, \\ 81310 UTM JB, Johor, Malaysia \\ ${ }^{3}$ School of Electronic, Electrical and Computer Engineering, University of Birmingham, Edgbaston, \\ B15 2TT Birmingham, United Kingdom \\ ${ }^{4}$ School of Electrical System Engineering, Universiti Malaysia Perlis, 02600 Arau, Perlis, Malaysia \\ kamilia@utm.my,mkamal@fke.utm.my, p.s.hall@bham.ac.uk, asmawati@fke.utm.my, ezwanjalil@gmail.com, \\ mfareq@unimap.edu.my
}

\begin{abstract}
Textile diamond dipole and Artificial Magnetic Conductor (AMC) have been proposed and tested under wearable and body centric measurements. The proposed antenna and AMC sheet are entirely made of textiles for both the substrate and conducting parts, thus making it suitable for wearable communications. Directive radiation patterns with high gain are obtained with the proposed AMC sheet, hence minimizing the radiation towards the human body. In this study, wearable and body centric measurements are investigated which include bending, wetness and Specific Absorption Rate (SAR). Bending is found not to give significant effect to the antenna and AMC performance, as opposed to wetness that yields severe performance distortion. However, the original performance is retrieved once the antenna and AMC dried. Moreover, notable SAR reduction is achieved with the introduction of the AMC sheet, which is appropriate to reduce the radiation that penetrates into human flesh.
\end{abstract}

\section{Keywords}

Textile antenna, diamond dipole, textile artificial magnetic conductor, bending, wetness, Specific Absorption Rate

\section{Introduction}

Wearable antennas experience performance degradation such as frequency detuning, bandwidth reduction and radiation distortions when placed on human body [1] Moreover, the radiation that penetrates into the human cells is a major health concern [2]. To overcome such issues, textile AMC sheet has been introduced which is flexible and suitable for wearable communications [3]. AMC surface helps by minimizing the radiation exposure to the human body, apart from enhancing the gain. Textile antenna and AMC sheet are meant for wearable application [2-6]. Human body is a complex and inhomogeneous environment for wearable antennas to perfectly operate. Furthermore, wearable antennas that are mounted on human body are subjected to dynamic environment changes such as movements and exposure to nature, e.g. rain and snow. Such environment variation is a challenge for textile antennas to function effectively [1]. Apart from the interaction between antenna and human body, the wearable antennas are exposed to several dynamic conditions including bending [7] and wetness [8]. Moreover, as mentioned earlier, wearable antennas that are placed very close to human body create health concerns regarding the penetration of electromagnetic radiation onto the human's flesh [9]. In this study, a textile diamond dipole with AMC ground plane is proposed. In addition, in order to test whether or not the performance of the wearable antenna and AMC are viable for body centric applications, this study will discuss the measurements conducted for the antenna and AMC, under bending and wet conditions. SAR investigation will also be presented at the end of this article.

\section{Textile Antenna with Artificial Magnetic Conductor}

This study concentrates on the development of antenna and AMC structures made of textile for wearable communication. The first section presents the design of textile diamond dipole antenna. Subsequently, the second section describes the design process of the textile AMC sheet, which involves unit cell characterization using tran- 
sient solver computed by Computer Simulation Technology (CST) software, prior to the AMC arrays design.

\subsection{Textile Diamond Dipole}

Diamond dipole antenna made of textile has been designed in this study. The antenna is to be incorporated with textile AMC structure that will be discussed in the next section. The main fabric used in this study is fleece with permittivity $\varepsilon_{\mathrm{r}}=1.3$, tangent loss $\delta=0.025$ and thickness $h=1 \mathrm{~mm}$. Shieldit fabric is used for the conducting parts of the dipole and AMC. Diamond dipole antenna is an inverted bow-tie antenna, and it is obtained by flipping the two halves of the bow-tie antenna [10]. Diamond dipole offers wider bandwidth compared to the conventional planar straight dipole [11], [12]. Based on the design in [13], a $2.45 \mathrm{GHz}$ textile diamond dipole is designed and the layout along with its dimensions is as shown in Fig. 1.

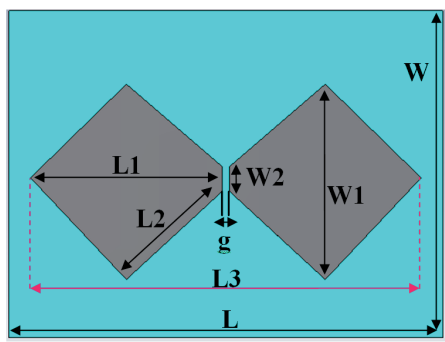

Fig. 1. Textile diamond dipole with its dimensions in $\mathrm{mm}$. $L=60, W=45, L 1=26.5, L 2=18, W 1=27, W 2=3.4, g=1$.

After the simulation process, fabrication of the antenna follows. The prototype of the textile diamond dipole is shown in Fig. 2. Pigtail SMA is used as the connector for the diamond dipole. This cable type connector is deemed to be suitable to be used in wearable application as opposed to the typical bottom fed SMA connector.

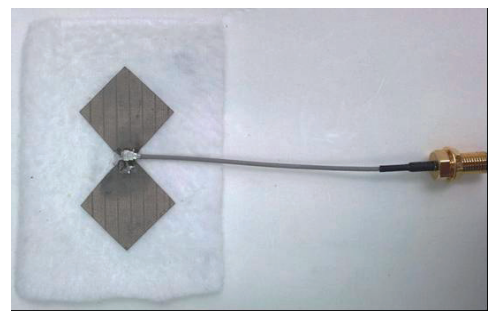

Fig. 2. Fabricated textile diamond dipole.

Balun is often needed to prevent return current from the third arm. Inner conductor of coaxial with certain length will act as the third arm of the radiator. In this work, balun is not considered because the influence of asymmetric feeder is negligible in a complex human body environment. In addition, comparison between simulated and measured results show that the feed is matched since the effective length of the feeder gives impedance matching of $50 \mathrm{ohm}$. As such, the overall size and cost of the antenna becomes lower without the addition of balun.
Measurements have been performed to validate the simulation findings. The measured $\mathrm{S}_{11}$ for the diamond dipole is plotted and compared with the simulation in Fig. 3. From the results, reasonable agreement between simulation and measurement can be seen. The measured $\mathrm{S}_{11}$ has a depth of $-33.64 \mathrm{~dB}$ with bandwidth of $750 \mathrm{MHz}$ (30.93\%) that ranging from $2.05 \mathrm{GHz}$ to $2.8 \mathrm{GHz}$. From the results in Fig. 3, it can be observed that there is slight discrepancy between simulated and measured return loss. The measured $S_{11}$ is found to yield higher return loss depth compared to the simulated value. However, measured result gives good resonance at $2.45 \mathrm{GHz}$, hence validating the simulation.

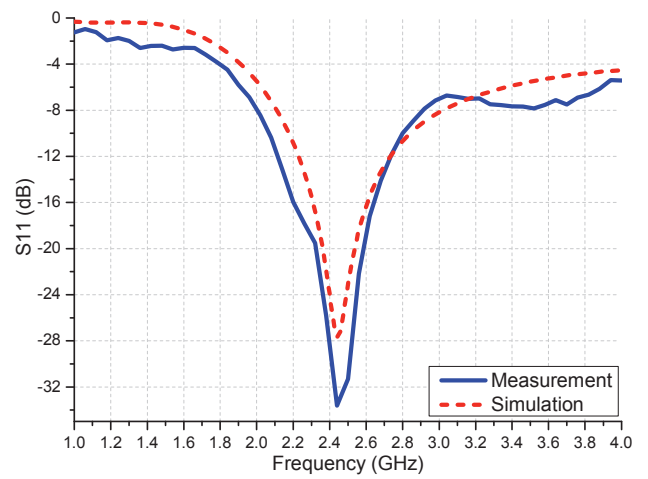

Fig. 3. Measured and simulated $\mathrm{S}_{11}$ of textile diamond dipole.

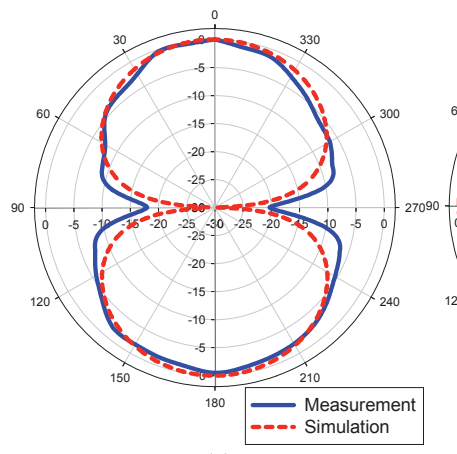

(a)

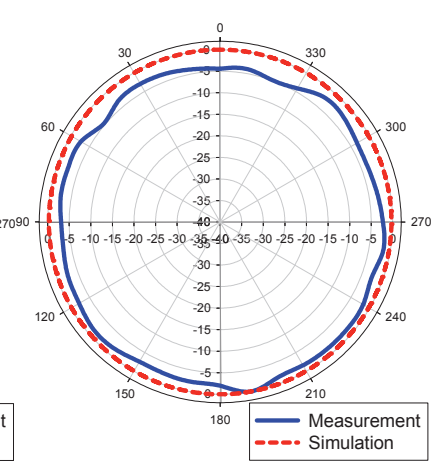

(b)
Fig. 4. Measured and simulated radiation patterns of textile diamond dipole at $2.45 \mathrm{GHz}$.

The radiation pattern measurement has been conducted in an anechoic chamber and the results are presented and compared with the simulation in Fig. 4. The measured patterns in $\mathrm{E}$ and $\mathrm{H}$ planes show good agreement with the simulation. The measured gain is $3.09 \mathrm{~dB}$ and $2.04 \mathrm{~dB}$ for the simulated value. The designed antenna has computed efficiencies of $98.77 \%$. Very close agreement has been obtained between the measurement and simulation in terms of resonance, bandwidth and radiation pattern. However, there is discrepancy between simulated and measured gain that is predicted due to dissimilarity between the simulated structure and fabricated prototype. The pigtail SMA connector is not included in the simulated structure to reduce simulation size and time, where discrete port has been simulated as the antenna excitation. 


\subsection{Textile Diamond Dipole above AMC}

Continuing from the textile antenna design, the development of textile AMC structure is then explored. In this study, textile AMC is designed to have in-phase reflection at $2.45 \mathrm{GHz}$. The AMC consists of conductive patches on a textile substrate that is backed with a ground plane. Similar to the antenna design, the substrate of the AMC is also made of fleece fabric whereas the conducting patches and ground plane are made of Shieldit fabric.

To design the AMC array, a unit cell characterization using CST transient solver is performed as shown in Fig. 5. The unit cell simulation represents infinite array by manipulating the boundary conditions in CST. Through unit cell simulation, computational time in designing an AMC arrays can be largely reduced. With boundary conditions assigned as perfect electric and perfect magnetic boundaries that are perpendicular to each other, an infinite array approach can be achieved. Plane wave is set to illuminate the surface of the AMC unit cell and the phase of the reflected wave is recorded to obtain the reflection phase graph.
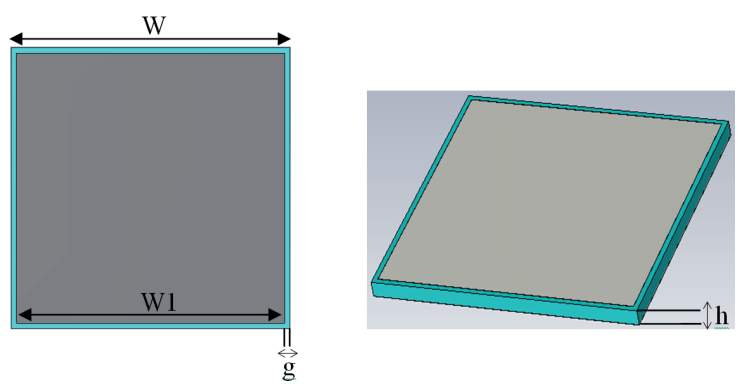

Fig. 5. Unit cell model of the textile square patch AMC with dimensions in mm. $W=52, W 1=50, g=1, h=1$.

Initially, equations (1-4) are referred, in order to have a rough parameters' estimation of the AMC unit cell. Based on AMC equivalent circuit derived in [14], the fringe electric field between adjacent metal patches creates the capacitance that is placed at a distance $h$ from a shortcircuited dielectric loaded transmission line. AMC can be modeled as a distributed LC circuit with typical high-impedance surface. The impedance of the structure is given by (1). The gaps between the metal patches indicate the level of capacitance, $C$ while the height of the substrate represents the inductance, $L$ [15]. $f_{\mathrm{c}} \varepsilon_{\mathrm{o}}, W, g, \mu_{\mathrm{o}}$ and $h$ denote the operating frequency, permittivity of free space, patch width, gap between patches, permeability of free space and substrate thickness respectively.

$$
\begin{gathered}
Z_{\text {in }}=\frac{j \omega L}{1-\omega^{2} L C}, \\
f_{r}=\frac{1}{2 \pi \sqrt{L C}}, \\
C=\frac{W \varepsilon_{0}\left(1+\varepsilon_{r}\right)}{\pi} \cosh ^{-1}\left(\frac{W+g}{g}\right), \\
L=\mu_{0} h .
\end{gathered}
$$

In this study, a simple geometry of square AMC structure is proposed due to the limitation of textile materials as well as constraints in fabrication. Such design is simple, low cost and easy to fabricate apart from giving satisfactory performance. Vias are not included in the AMC design, for simplicity and easy fabrication. Following the numerical calculation, unit cell simulation is conducted to investigate the reflection phase characteristics of a square textile AMC. Figure 6 illustrates the optimized reflection phase diagram of a fleece square patch with $50 \mathrm{~mm}$ width resonating at $2.45 \mathrm{GHz}$.

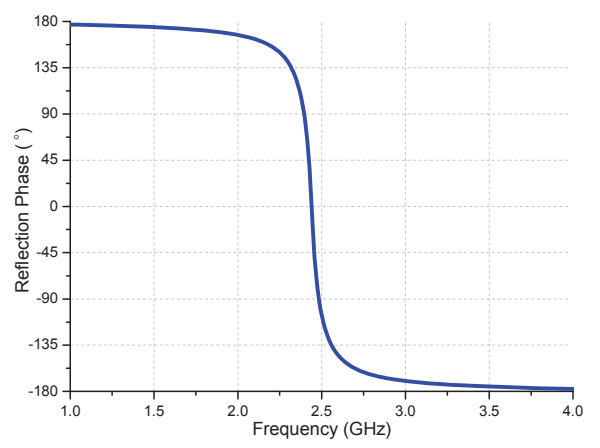

Fig. 6. Optimized reflection phase diagram of a $2.45 \mathrm{GHz}$ textile square patch AMC.

From the unit cell simulation, AMC arrays are then subsequently designed and the antenna performance above the structure is investigated. The fleece AMC comprises of $6 \times 4$ conductive patches backed with a ground plane. After optimization, the patch size is $51 \mathrm{~mm}$ with $2 \mathrm{~mm}$ gap between the patches. Figure 7 shows the fabricated textile $\mathrm{AMC}$ with the diamond dipole. The dimension of the AMC arrays is $318 \mathrm{~mm} \times 212 \mathrm{~mm}$. A special cutter machine is used to fabricate the AMC patches for better accuracy. The antenna is placed $5 \mathrm{~mm}$ above the AMC surface. The $5 \mathrm{~mm}$ distance is maintained by using a special material, Rohacell $31 \mathrm{HF}$ with dielectric constant of 1.05 . This material is used as the spacer between the antenna and the AMC surface. Such material has permittivity that is very close to air which is suitable to replace the air gap in the simulation.

In this study, investigations for both free space and on-body environments are considered. Figure 7(b) shows the on-body measurement setup for the textile diamond dipole above the AMC sheet. Vector network analyzer is used to measure the S-parameter performance. The antenna

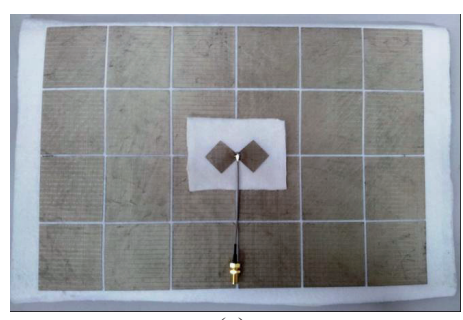

(a)

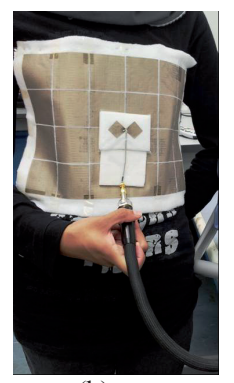

(b)
Fig. 7. Diamond dipole above AMC sheet: (a) fabricated prototype; (b) on-body measurement. 
and AMC sheet are meant to be worn around the torso area hence they are positioned at the centre of the subject's chest. The AMC sheet is closely attached to the subject's body to minimize the air gap. Furthermore, the wearer's hand is ensured to be placed further from the antenna and AMC during measurement for accurate measurement by avoiding unwanted effect from the human's hand.

Figure 8 shows the simulated and measured $\mathrm{S}_{11}$ of the diamond dipole above textile AMC structure. Measured result shows reasonable agreement with the simulation with resonance achieved at $2.45 \mathrm{GHz}$. Simulated return loss at $2.45 \mathrm{GHz}$ is $-19.54 \mathrm{~dB}$ with bandwidth of $6.92 \%$ from $2.37 \mathrm{GHz}$ to $2.54 \mathrm{GHz}$. On the other hand, measured reflection coefficient depth is $-23.09 \mathrm{~dB}$ with bandwidth ranging from $2.17 \mathrm{GHz}$ to $2.57 \mathrm{GHz}$ giving a bandwidth of $16.8 \%$.

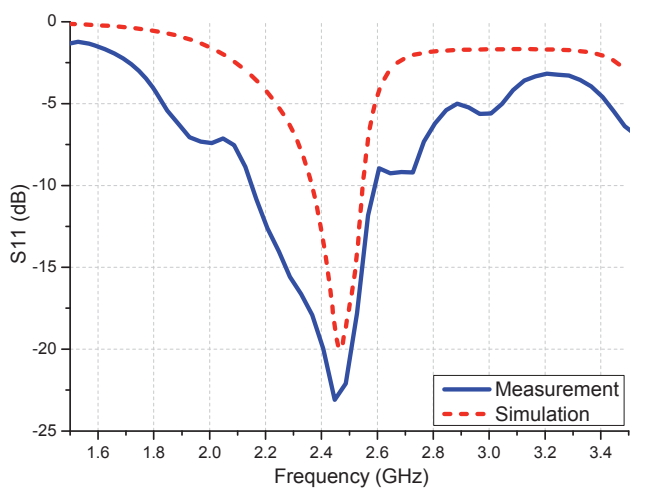

Fig. 8. Measured and simulated $\mathrm{S}_{11}$ of diamond dipole above AMC sheet.

Figure 9 shows the measured reflection coefficient of the diamond dipole in on-body environment. The graph compares the $\mathrm{S}_{11}$ of diamond dipole alone and when placed above textile AMC sheet. A shift of resonant frequency to 2.27 GHz is observed when the diamond dipole is placed on human body. When placing the antenna above the AMC sheet, the resonant frequency shifts to $2.37 \mathrm{GHz}$. Despite the shift in resonance, the antenna is still performing well at $2.45 \mathrm{GHz}$ with return loss of $-13.44 \mathrm{~dB}$ for the antenna alone and $-15.09 \mathrm{~dB}$ for the case with the presence of AMC sheet.

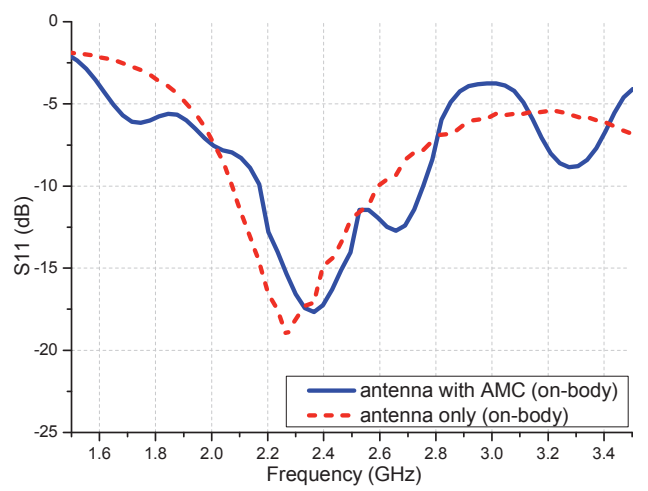

Fig. 9. Measured $S_{11}$ of diamond dipole with and without $\mathrm{AMC}$ in on-body environment.

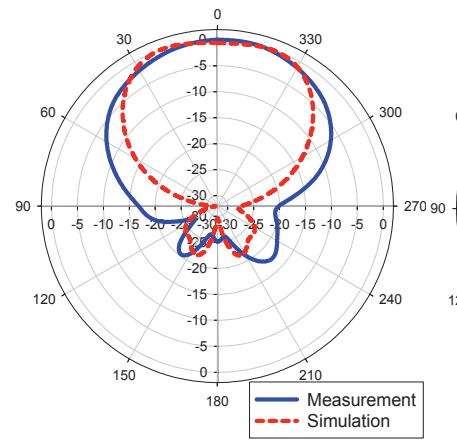

(a)

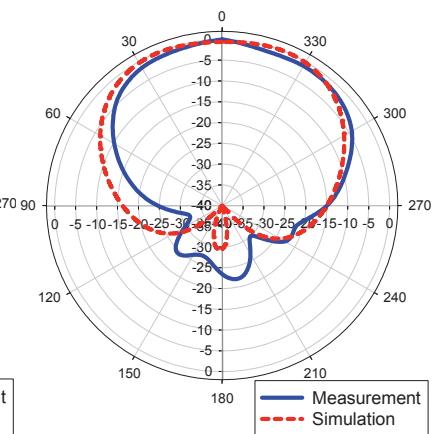

(b)
Fig. 10. Measured radiation patterns of textile diamond dipole above $\mathrm{AMC}$ at $2.45 \mathrm{GHz}$ : (a) E plane, (b) H plane.

The simulated and measured radiation patterns in $\mathrm{E}$ and $\mathrm{H}$ planes are shown in Fig. 10. Measured results agree well with the simulated results, giving directive patterns with small back lobes. Such forward directive pattern is appropriate for wearable application since it minimizes the radiation that penetrates into the human body. The simulated gain of the antenna above AMC sheet is observed as $5.43 \mathrm{~dB}$; and $2.1 \mathrm{~dB}$ without the AMC surface. The measured gain gives similar trend with $6.53 \mathrm{~dB}$ for antenna with AMC and $3.09 \mathrm{~dB}$ for just the antenna. With the introduction of AMC sheet, significant gain improvement is expected due to the AMC in-phase reflection properties.

\section{Wearable and Body Centric Measurements}

This section explores the effect of textile antenna and AMC under wearable and body centric measurements. Three types of measurements are conducted which include bending, wetness and SAR to test the viability of the proposed textile antenna and AMC for wearable application.

\subsection{Bending}

In body centric environment, the performance of antenna under bending condition is a crucial factor to consider. Since textile antenna is designed for wearable application, it is subjected to the change of human body's posture as well as its movements. Therefore, the antenna is prone to bending and it is difficult to maintain the antenna in a flat form. Due to that, bending measurement is necessary to be carried out.

To demonstrate bending condition in human body, polystyrene cylinders are used as the bending set up. The relative permittivity of the polystyrene is 1.06 , which is close to the permittivity of air. In this study, bending measurement is performed for the case of antenna on its own and for antenna with AMC. For the case of antenna only, small polystyrene cylinder that represents a human arm size with diameter of $80 \mathrm{~mm}$ has been used. On the other hand, two polystyrene cylinders with diameter of $250 \mathrm{~mm}$ and $310 \mathrm{~mm}$ that mimic the size of small torso and large 


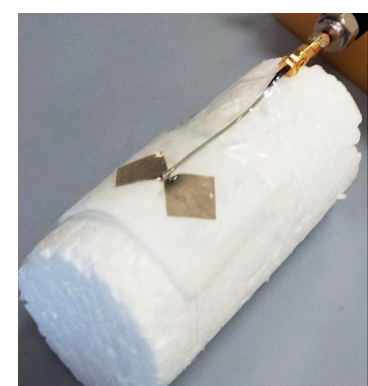

(a)

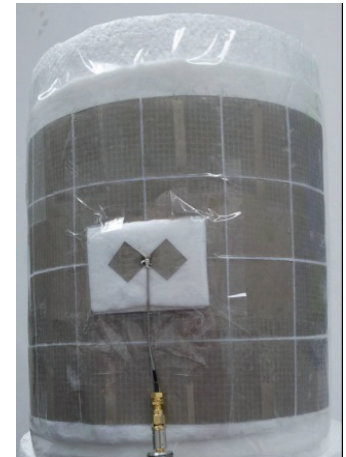

(b)
Fig. 11. Bending measurement setup: (a) antenna only, (b) antenna with AMC.

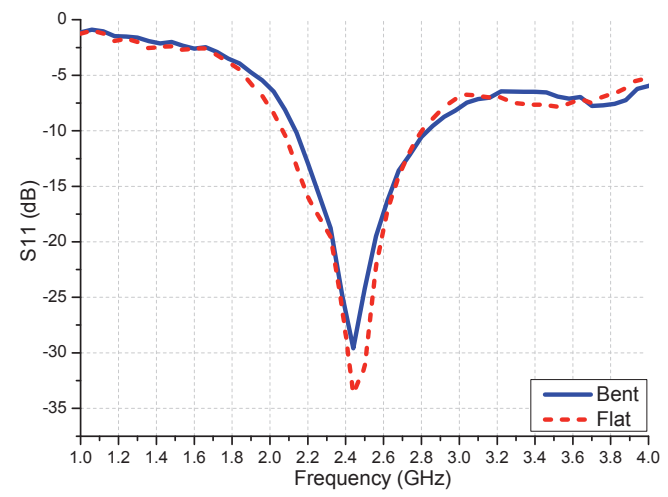

Fig. 12. Measured $S_{11}$ of textile diamond dipoles under bending condition.

torso are used for antenna and AMC bending measurements. The bending measurement setup is shown in Fig. 11. In this study, the textile diamond dipole is bent in horizontal orientation as shown in the figure. Thin sellotape is used to fix the position of the antenna accordingly.

Figure 12 depicts the measured reflection coefficient of a bent versus a flat textile diamond dipole. From the result, reasonable agreement can be seen between the two curves. The resonant frequency is observed to remain stable even with bending. The reflection coefficient depth for the bent textile diamond dipole is found to decrease from $-33.6 \mathrm{~dB}$ to $-29.6 \mathrm{~dB}$. However, generally the resonant frequency and impedance bandwidth for the bent case is found to yield a good performance for the textile diamond dipole. The measured result shows that the textile antenna exhibits good performance under bending state. The small size of the antenna in comparison to the cylinder's size might result to the negligible changes to the impedance matching.

The radiation pattern of the bent diamond dipole is shown in Fig. 13. The radiation patterns are seen to have good agreement between the bent and flat conditions. The radiation characteristics with shape of a dipole are retained. However from observation, the beamwidths are slightly widened under bending. The bending curvature has broadened the direction of the antenna hence resulting the antenna to have a slightly higher beamwidth. The measured gain for the bent dipole is $2.63 \mathrm{~dB}$ as compared to $3.09 \mathrm{~dB}$

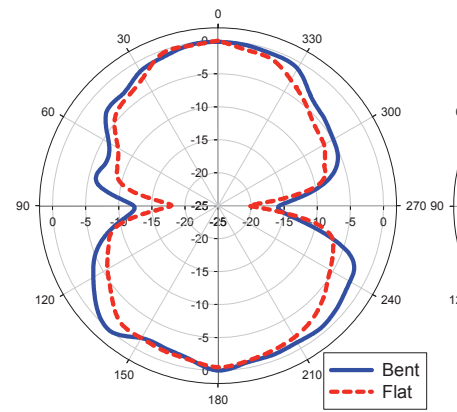

(a)

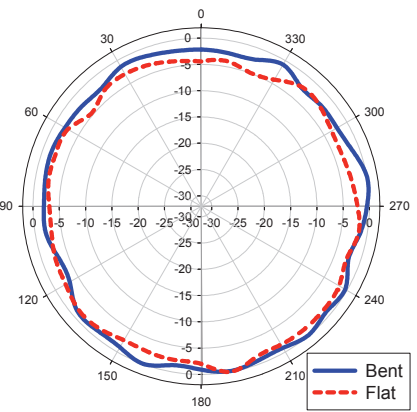

(b)
Fig. 13. Measured radiation patterns of textile diamond dipole under bending condition: (a) E plane, (b) H plane.

for the flat antenna. The measured gain is observed to slightly drop for the bent antenna. The small drop is expected since bending has caused the antenna to be less directional and therefore a drop of gain is seen.

Next, the bending experiment is carried out for the textile antenna with AMC case. Figure 14 shows the measured return loss of bent diamond dipole with $\mathrm{AMC}$ sheet. The graph compares the $\mathrm{S}_{11}$ of a flat versus bent configurations, i.e. small and large bending. A good agreement is observed between the bent and flat cases. Results show that bending on a small cylinder exhibits a higher deviation in reflection coefficient, compared to a large cylinder due to a higher degree of bending which results in a more apparent deviation. In addition, the size of the AMC sheet is relatively large; hence a bending exposure is more noticeable than the previous case of antenna only.

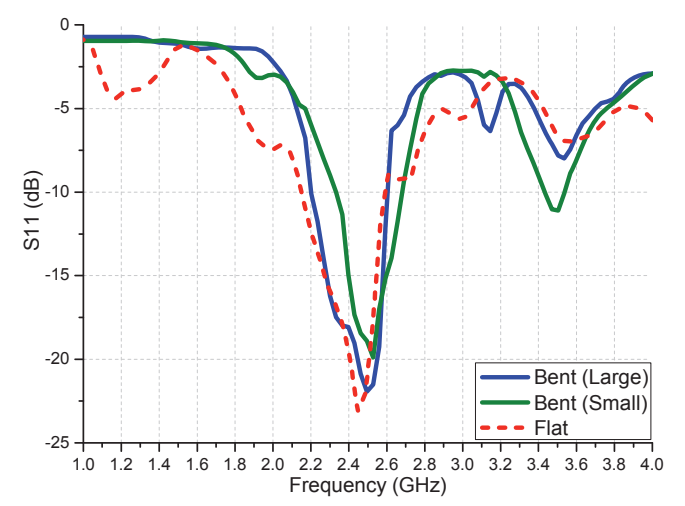

Fig. 14. Measured $S_{11}$ of textile diamond dipole above AMC under bending condition.

Polar plots in Fig. 15 show the radiation patterns of textile diamond dipole above AMC sheet in bent and flat conditions. For radiation pattern and gain measurements, only smaller cylinder of size $250 \mathrm{~mm}$ is used. Radiation pattern results show a reasonable agreement between bent and flat cases. Similar as flat configuration, the bent antenna and AMC also obtain directional patterns with small backlobes. Since the antenna and AMC are being bent on a curvature, they are forced to face broader direction, hence they become less directional. Therefore, higher backlobes are observed for the bent cases in both planes.

When the diamond dipole with AMC sheet is bent, the measured gain is $5.83 \mathrm{~dB}$. The gain experiences small 


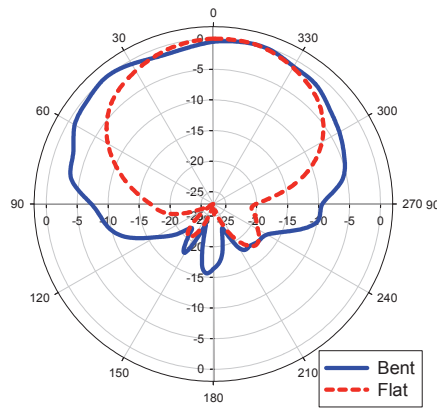

(a)

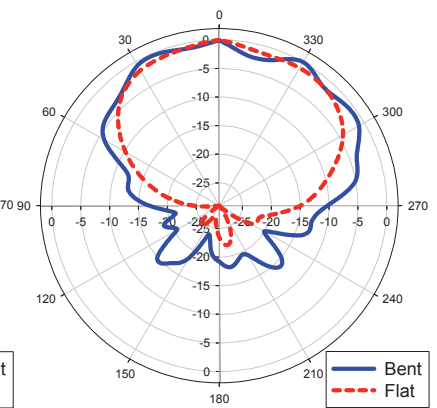

(b)
Fig. 15. Measured radiation patterns of textile diamond dipole above AMC sheet under bending condition at $2.45 \mathrm{GHz}$ : (a) E plane, (b) H plane.

drop of $0.7 \mathrm{~dB}$ from $6.53 \mathrm{~dB}$ for the flat configuration. Since the bent configuration has become less directional, the drop of gain is expected. Nonetheless, the bent configuration of diamond dipole and AMC still exhibits directional pattern with a small backward radiation along with significantly high gain.

\subsection{Wetness}

The proposed textile antenna and AMC are designed to be integrated into human clothing for daily wear. Hence, there is a tendency for the antenna and AMC to get wet due to sweat or rain. Although the textile antenna can be designed with a waterproof plastic coating, typical fabric clothing materials are not waterproof, and therefore wetness test is necessary. Water has a high relative permittivity of approximately 80 . When a textile antenna becomes wet, the water that penetrates into the fabric dominates the dielectric constant of the antenna's substrate. Therefore, a wet antenna consequently has higher relative permittivity compared to when it is in a dry state. The presence of water changes the properties and parameters of the textile antenna. This will in turn change the performance of the antenna, particularly the resonant frequency. Due to this reason, this section will investigate the performance of textile antenna and AMC under wet condition. Similar as in the previous bending study, wetness measurements will also be conducted for two cases i.e. antenna only, and antenna with AMC.

Figure 16 shows the textile diamond dipole and AMC sheet being immersed inside the water for the wetness measurement. After being soaked overnight for more than

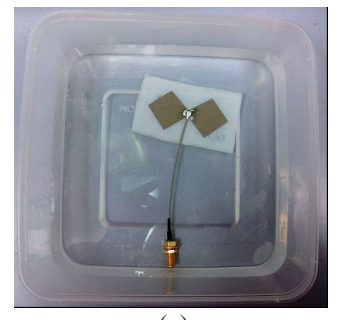

(a)

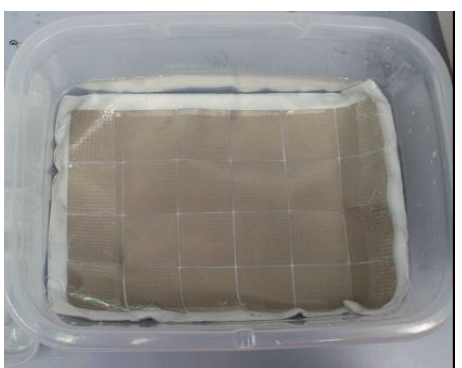

(b)
Fig. 16. Wetness measurement: (a) antenna, (b) AMC sheet.
12 hours, the return loss of the antenna is measured in a complete wet state. By using the Vector Network Analyzer, $\mathrm{S}_{11}$ of the wet antenna is measured immediately after being taken out from the water.

The return loss comparison between different wetness conditions is plotted in Fig. 17. The $\mathrm{S}_{11}$ results are being compared in four conditions namely before washing, complete wet, damp and dry. The dashed red line represents the $\mathrm{S}_{11}$ of completely wet textile diamond dipole. As expected, a severe distortion has been achieved. The high permittivity of water influences the antenna performance.

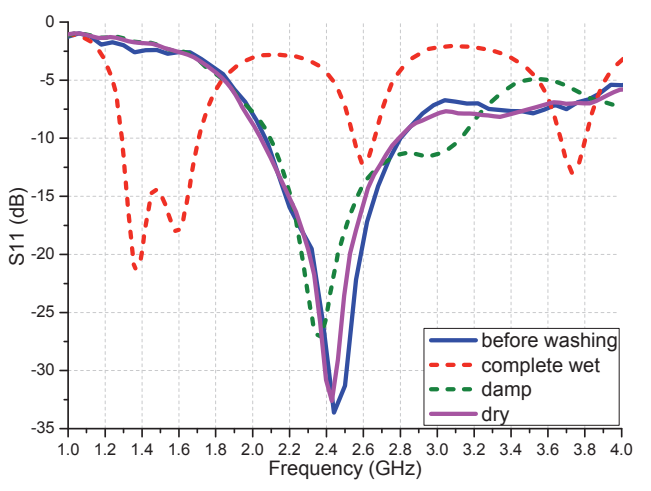

Fig. 17. Measured $S_{11}$ of textile diamond dipole under wet condition.

As can be seen from the graph, the resonant frequency has been shifted to lower frequency since the wet antenna substrate has high dielectric constant. For the damp case, the return loss curve has returned close to the original resonant frequency. The resonant frequency for damp diamond dipole is $2.37 \mathrm{GHz}$ with return loss depth of $-27.3 \mathrm{~dB}$. Although the damp antenna is almost dry, some moisture is still left inside the antenna. Therefore, its dielectric constant is still relatively higher than the actual original value. The fully dried $\mathrm{S}_{11}$ curve is denoted by the pink line in Fig. 17. The resonant frequency for the fully dried diamond dipole is $2.43 \mathrm{GHz}$ with return loss depth of $-32.6 \mathrm{~dB}$. It can be observed that there is slight deviation between the dry and original antennas. This is due to the fabric shrinking that changes the original properties of the antenna. Nevertheless, the fully dried antenna still exhibits good performance as offered by the original before washing diamond dipole.

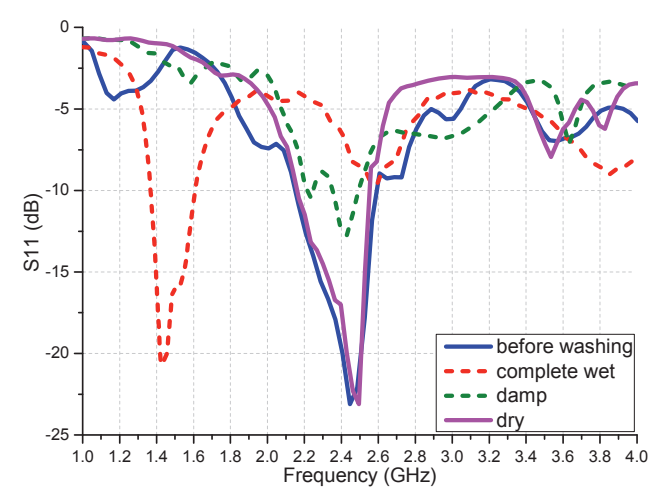

Fig. 18. Measured $S_{11}$ of textile diamond dipole above AMC under wet condition. 
The wetness measurement is then continued for the case of antenna with AMC. The measured return loss of the diamond dipole and AMC is shown in Fig. 18 which compares the $\mathrm{S}_{11}$ results for the states of before washing, complete wet, damp and dry. Since the wet antenna and AMC are completely influenced by the high permittivity of water, dramatic distortion is observed. The high permittivity of water corresponds to a lower resonance as shown by the dashed red curve. The antenna and AMC are then dried and the damp result shows that the $\mathrm{S}_{11}$ is slowly returning to the original result. Moisture still exists in the antenna and $A M C$; hence the $S_{11}$ result is still not exactly similar as the original's result. Finally, once the configuration is completely dry, a good agreement of return losses between the before washing and dry are attained. Although there is a slight deviation, a satisfactory result has been achieved once the antenna and AMC return to dry state.

\subsection{Specific Absorption Rate}

The proposed textile antenna and AMC sheet will be applied for on-body communication. For body centric applications, the antenna and AMC need to be placed close to human body. Therefore, Specific Absorption Rate (SAR) values are crucial for evaluation. SAR is a measure of the quantity of Radio Frequency (RF) energy that is absorbed by a unit mass of body. SAR value is expressed in unit of Watts per kilogram $(\mathrm{W} / \mathrm{kg})$. The equation that governs SAR value is as shown below (5). $E$ is total field strength $(\mathrm{V} / \mathrm{m}), \sigma$ is conductivity $(\mathrm{S} / \mathrm{m})$ and $\rho$ is material mass density $\left(\mathrm{kg} / \mathrm{m}^{3}\right)$.

$$
S A R=\frac{\sigma|E|^{2}}{\rho}(\mathrm{W} / \mathrm{kg}) .
$$

SAR simulation is performed using CST Microwave Studio which offers inhomogeneous biological models called CST Voxel family. In this research, Gustav, a 38 years old male with complete and detailed organ model, was used. Gustav model is a $176 \mathrm{~cm}$ tall male with weight of $69 \mathrm{~kg}$. The model has conductivity $\sigma$ and permittivity $\varepsilon_{\mathrm{r}}$ that comply with the standards tissue dielectric parameters recommended by the Federal Communications Commission (FCC). Limits of SAR have been imposed by FCC [16] in the US and by International Commission on NonIonizing Radiation Protection (ICNIRP) [17] for the worldwide. SAR limit enforced by FCC is $1.6 \mathrm{~W} / \mathrm{kg}$ for a mass of 1 gram and ICNIRP's limit is $2 \mathrm{~W} / \mathrm{kg}$ for 10 grams of tissues.

Simulations are conducted for diamond dipole with and without AMC surface placed on the torso part of Voxel biological body. Since the antenna and AMC groundplane are to be worn around the torso area, the dipole is placed at the centre of the phantom's chest. Reference power of $1 \mathrm{~W}$ is excited for the simulation and the SAR investigation is performed at $2.45 \mathrm{GHz}$.

$3 \mathrm{D}$ visualization of SAR is illustrated in Fig. 19. Figures show the 3D plot with information of maximum SAR

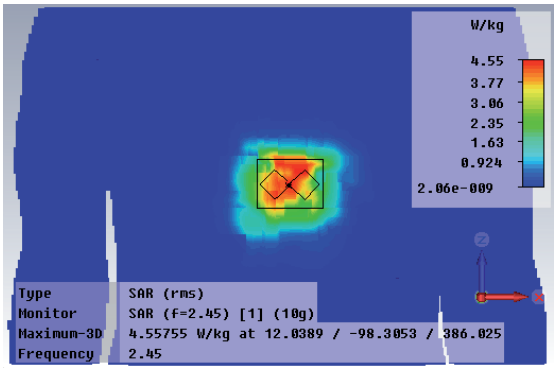

(a)

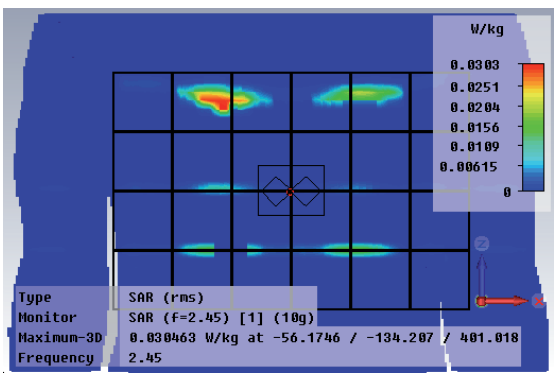

(b)

Fig. 19. Simulated 3D maximum SAR visualization in CST: (a) antenna only, (b) antenna with AMC.

position. From the figures, it is clearly shown that high concentration of SAR value is seen when the textile antenna is placed alone on the human body. On the other hand, very minimum SAR concentration is observed when the textile AMC waveguide sheet is placed beneath the antenna. From the simulated 3D SAR diagrams, the maximum 3D $10 \mathrm{~g}$ SAR value for textile diamond dipole alone is $4.56 \mathrm{~W} / \mathrm{kg}$. On the contrary, the peak 3D $10 \mathrm{~g} \mathrm{SAR}$ for the antenna with AMC surface is $0.03 \mathrm{~W} / \mathrm{kg}$. From the results, it can be seen that the peak SAR value decreases significantly with the introduction of AMC sheet. In addition, the antenna's efficiency experiences a notable drop of $37 \%$ with the existence of the phantom. However, the influence of the phantom towards the efficiency lessens with a fall of $3.5 \%$ when having AMC surface.

The SAR measurement is conducted using a simple system provided by Indexsar. IXP-050 SAR probe and Probe Amplifier IXA-020 are used in this research. The probe covers range of $400 \mathrm{MHz}$ to $6 \mathrm{GHz}$. However, in this work, only performance at $2.45 \mathrm{GHz}$ will be investigated. The probe has been calibrated by Indexsar in air, brain and body liquids. Waveguide method is employed for rotational isotropy calibrations. The probe is built with dipole sensors that are placed on a triangular prism core. The probe is tested to be resistant to glycol and liquid containing alcohol. Accompanying software that extracts the measured E fields and numerically processes and displays the SAR values are also provide by Indexsar.

Tissue stimulant liquid used in this work is also provided by Indexsar. The phantom tissue stimulant liquid operates at $2.45 \mathrm{GHz}$. The liquid contains Polyoxyethylene Sorbitan Aliphatic Acid mixture, which is a non toxic and does not contain Glycol. The measured dielectric properties of the liquid are 53.57 for the permittivity $\varepsilon_{\mathrm{r}}$ and 1.81 for 


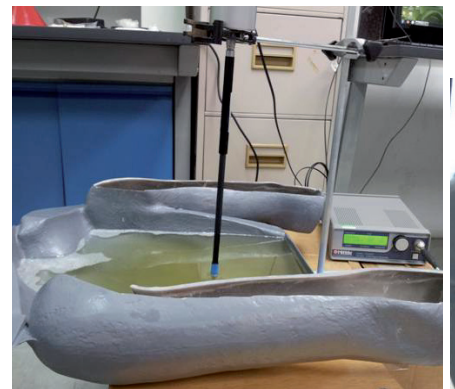

(a) (b)

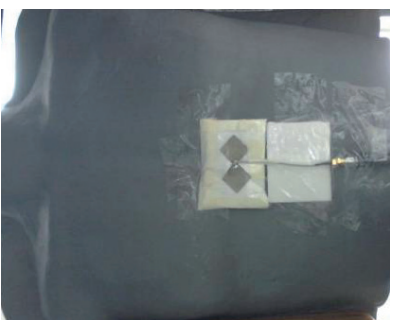

Fig. 20. SAR measurement setup using Indexsar probe and tissue stimulant liquid with human phantom body made of fibre glass: (a) side view, (b) bottom view.

the conductivity $\sigma$ at $22^{\circ} \mathrm{C}$. On the other hand, due to the high cost, the phantom body used in this measurement setup is not provided by Indexsar. A human phantom for the torso body part has been fabricated separately using fibreglass.

Figure 20 shows the SAR measurement setup using Indexsar probe and software together with the fibreglass phantom for torso part. $2.45 \mathrm{GHz}$ equivalent homogeneous tissue stimulant liquid is filled into the phantom body. The probe is immersed in the liquid. When antenna is excited to the phantom body, the probe will detect the measured data and send it to the software to calculate and display the peak point SAR value.

Measurement is conducted to investigate the SAR performance of the textile diamond dipole with and without AMC sheet. Figure 21 compares the simulated value with the measured peak point SAR of the diamond dipole configurations. Results reveal that with the introduction of AMC sheet, peak point SAR is dropped to $0.037 \mathrm{~W} / \mathrm{kg}$ for the simulation and $0.033 \mathrm{~W} / \mathrm{kg}$ for the measured value. On the other hand, when the AMC structure is not present, the peak point SAR is as high as $26.4 \mathrm{~W} / \mathrm{kg}$ for simulation and $14.75 \mathrm{~W} / \mathrm{kg}$ for measurement. Significant drops of $26.36 \mathrm{~W} / \mathrm{kg}$ and $14.72 \mathrm{~W} / \mathrm{kg}$ are achieved for simulation and measurement respectively.

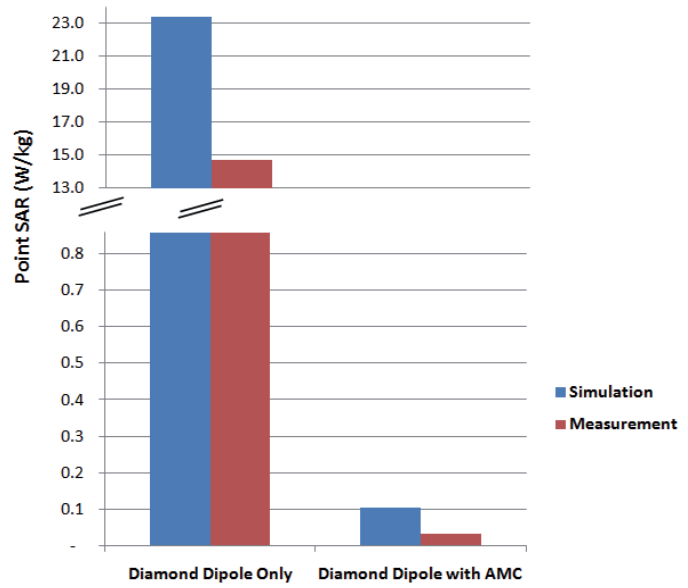

Fig. 21. SAR comparison between simulated and measured results for diamond dipole antenna with and without AMC sheet.
Following that, investigations on the separation distances between the antenna and AMC to the biological point in a human body are explored. The antenna and AMC are placed very close to the surface of human body. From there, the point SAR at different distances in the human body is evaluated at $0,10,20$ and $50 \mathrm{~mm}$. $0 \mathrm{~mm}$ denotes the nearest point to the excitation while $50 \mathrm{~mm}$ is the furthest point into the human body. Figure 22 shows the visual description of the mentioned arrangement.

Figure 23(a) plots the peak point SAR values in a function of distance for simulated and measured diamond dipole. The trend of the measured and simulated values shows good agreement. From the results, it shows that the peak point SAR values for both simulation and measurement decrease as the distance increases. On the other hand, Figure 23(b) depicts the peak point SAR versus distance results for diamond dipole with AMC configuration.

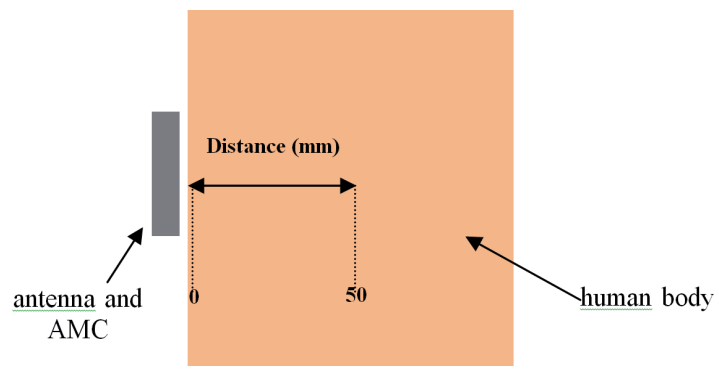

Fig. 22. Visual illustration of the distance between antenna and human body.

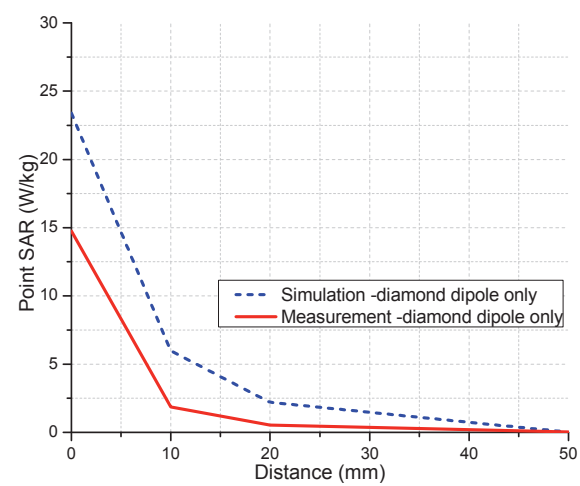

(a)

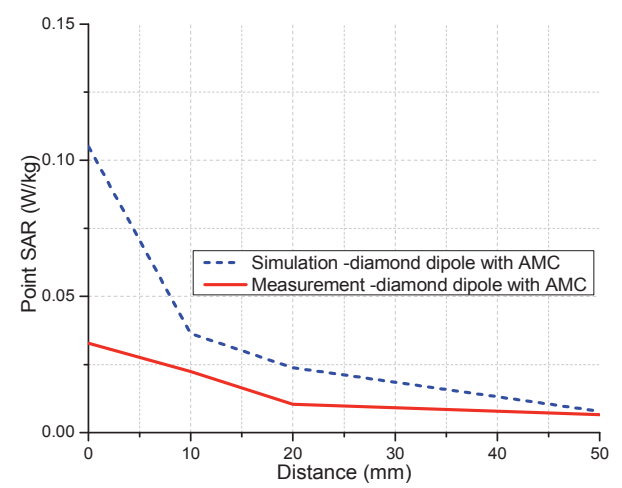

(b)

Fig. 23. Measured and simulated peak point SAR versus distance: (a) antenna only, (b) antenna with AMC. 
Similarly, peak point SAR values drop with an increment of distance. Reasonable agreement for the trend between measured and simulated results is also observed for the diamond dipole with AMC surface configuration. In addition, by comparing Fig. 23(a) and (b), significant drop of SAR values can be observed with the introduction of AMC ground plane. Measured results prove that SAR penetration can be reduced substantially by having AMC sheet.

From the presented results, good agreement in terms of trend has been achieved between simulation and measurement. However, there are discrepancies for the amplitude of the measured SAR compared to the simulation. The measured point SAR value is found to be smaller than the simulated values. The difference is expected since the outer dielectric shell for the fibreglass phantom used in the measurement is not model in the simulation. In the simulation, only biological human model is simulated with the antenna and AMC surface. Therefore, a high SAR value is expected due to the close proximity of excitation to the body surface. Due to that, small variations in the distance between the antenna and human body can lead to significant changes in SAR values. In addition, SAR measurement is often associated with uncertainty hence discrepancies between simulation and measurement can normally occur. Despite the difference of SAR amplitude between measured and simulated results, the trend shows good agreement as mentioned earlier. Furthermore, the AMC sheet has been proven to significantly reduce the SAR values. With AMC sheet, very low SAR value has been achieved hence enhancing its functionality for human health safety. Low SAR value proves that the amount of electromagnetic field being absorbed by the human body has been significantly reduced with the proposed AMC surface, which is appealing for health concern.

\section{Conclusion}

This study explored the development of textile diamond dipole with AMC sheet. With the introduction of the AMC ground plane, directive radiation patterns with a high gain were obtained. Reduced backlobe patterns which minimize the radiation that penetrates into the human body were observed. The proposed textile antenna and AMC were also tested under several wearable and body centric measurements. The impacts of bending, wetness and SAR have been studied rigorously for the cases of antenna only and antenna with AMC sheet. From the bending measurement, it is shown that bending does not give significant impact to the S-parameter, radiation pattern and gain performances. On the other hand, wetness investigation shows that a completely wet antenna and AMC suffer severe distortion in terms of S-parameter results. However, the performance of the antenna and AMC returned to its original before washing state when they are fully dried. Wetness can be avoided by laminating the textile antenna and AMC with a plastic coating or by using waterproof fabrics. SAR measurements have also been conducted for the de- signed textile antenna and AMC. Results show that significant reduction of SAR value is achieved with the presence of AMC sheet. The body centric measurements demonstrate that the proposed textile antenna and AMC sheet are deemed fit to be applied for wearable body centric application. However, the antenna and AMC are suggested to be coated with plastic cover to avoid any distortion caused by wetness. In addition, textile antenna and AMC with embroidered patches are also appealing for future work.

\section{Acknowledgments}

The authors wish to thank Malaysian Ministry of Science Technology and Innovation (MOSTI), Universiti Teknologi Malaysia (UTM) and Ministry of Education (MOE) for providing the Research Grant (Vote No: R.J130000.7823.4F277 and Q.J130000.2523.04H23). The authors also wish to thank Communications Engineering groups at the University of Birmingham and Universiti Malaysia Perlis for the help, guidance and facilities.

\section{References}

[1] HALL, P. S., HAO, Y. Antennas and Propagation for Body Centric Communications Systems. $2^{\text {nd }}$ ed. London (UK): Artech House, 2012, p. 63-64. ISBN: 1608073769

[2] ZHU, S., LANGLEY, R. Dual-band wearable antennas over EBG substrate. Electronic Letter, 2007, vol. 43, no. 3, p. 141-142. ISSN: 0013-5194. DOI: 10.1049/el:20073151

[3] SALONEN, P., YANG, F., RAHMAT-SAMII, Y., KIVIKOSKI, M. WEBGA - Wearable Electromagnetic Band-Gap Antenna. In IEEE International Symposium on Antennas and Propagation. 2004, p. 451-454. DOI: 10.1109/APS.2004.1329671

[4] MANTASH, M., TAROT, A.-C., COLlARDEY, S., MAHDJOUBI, K. Investigation of flexible textile antennas and AMC reflectors. International Journal of Antennas Propagation, 2012, vol. 2012, p. 1-10. DOI: 10.1155/2012/236505

[5] SOH, P. J., VANDENBOSCH, G. A. E., OOI, S. L., RAIS, N. H. M. Design of a broadband all-textile slotted PIFA. IEEE Transactions on Antennas and Propagation, 2012, vol. 60, no. 1, p. 379-384. ISSN: 0018-926X. DOI: 10.1109/TAP.2011.2167950

[6] ROH, J.-S., CHI, Y.-S., LEE, J.-H., TAK, Y., NAM, S., KANG, T.J. Embroidered wearable multiresonant folded dipole antenna for FM reception. IEEE Antennas and Wireless Propagation Letters, 2010, vol. 9, p. 803-806. ISSN: 1536-1225. DOI: $10.1109 /$ LAWP.2010.2064281

[7] SUBRAMANIAM, S., GUPTA, B. Design and development of body-worn applications and its performance study under flat and bent positions. Microwave and Optical Technology Letters, 2011, vol. 53, no. 9, p. 2004-2011. DOI: 10.1002/mop.26188

[8] OSMAN, M. A. R., RAHIM, M. K. A., SAMSURI, N. A., ELBASHEER, M. K., ALI, M. E. Textile UWB antenna bending and wet performances. International Journal of Antennas and Propagation, 2012, vol. 2012, p. 1-12. DOI: $10.1155 / 2012 / 251682$

[9] CHAHAT, N., LEDUC, C., ZHADOBOV, M., SAUlEAU, R. Antennas and interaction with the body for body centric wireless 
communications at millimeter waves. In 7th European Conference on Antennas and Propagation (EuCAP). Gothenburg (Sweden), 2013, p. 772-775.

[10] GHOSH, D., DE, A., TAYLOR, M. C., SARKAR, T. K., WICKS, M. C., MOKOLE, E. L. Transmission and reception by ultrawideband (UWB) antennas. IEEE Antennas and Propagation Magazine, 2006, vol. 48, no. 5, p. 67-99. ISSN: 1045-9243. DOI: 10.1109/MAP.2006.277157

[11] PERRUISSEAU-CARRIER, J., HEE, T. W., HALL, P. S. Dualpolarized broadband dipole. IEEE Antennas and Wireless Propagation Letters, 2003, vol. 2, no. 1, p. 310-312. ISSN: 15361225. DOI: 10.1109/LAWP.2004.824165

[12] AKHOONDZADEH-ASL, L., KERN, D. J., HALL, P. S., WERNER, D. H. Wideband dipoles on electromagnetic bandgap ground planes. IEEE Transactions on Antennas and Propagation, 2007, vol. 55, no. 9, p. 2426-2434. ISSN: 0018-926X. DOI: 10.1109/TAP.2007.904071

[13] JALIL, M.E., RAHIM, M.K.A., SAMSURI, N.A., MURAD, N.A., OTHMAN, N., MAJID, H.A. On-body investigation of dual band diamond textile antenna for wearable applications at $2.45 \mathrm{GHz}$ and $5.8 \mathrm{GHz}$. In 7 th European Conference on Antennas and Propagation (EuCAP). Gothenburg (Sweden), 2013, p. 414-417.

[14] AKHOONDZADEH-ASL, L. , NOURINIA, J., GHOBADI, C., HALL, P.S. Influence of element shape on the bandwidth of artificial magnetic conductors. Journal of Electromagnetic Waves and Applications, 2007, vol. 21, no. 7, p. 929-946. DOI: $10.1163 / 156939307780748995$

[15] KERN, D. J., WERNER, D. H., MONORCHIO, A., LANUZZA, L., WILHELM, M. J. The design synthesis of multiband artificial magnetic conductors using high impedance frequency selective surfaces. IEEE Transactions on Antennas and Propagation, 2005, vol. 53, no. 1, p. 8-17. ISSN: 0018-926X. DOI: $10.1109 /$ TAP. 2004.840540

[16] KWOK, C., CLEVELAND, R. F. Jr., MEANS, D. L. Evaluating Compliance with FCC Guidelines for Human Exposure to Radiofrequency Electromagnetic Fields Supplement C (datasheet). Federal Communications Commission Office of Engineering \& Technology, 42 pages. [Online]. Cited 1997-12-01. Available at: https://www.comsearch.com/articles/oet65c.pdf

[17] International Commission on Non-Ionizing Radiation Protection (ICNIRP). Guidelines for Limiting Exposure to Time-Varying Electric, Magnetic and Electromagnetic. Health Physics, 1998, vol. 74, no. 4, p. 494-522, ISSN: 0017-9078.

\section{About the Authors ...}

Kamilia KAMARDIN was born in 1982 in Johor, Malaysia. She received her B.Eng. Electronic (Communications) from the University of Sheffield, U.K., in 2004 and her M.Sc. from Universiti Teknologi Mara (UiTM), Malaysia in 2007. She obtained her Ph.D. in Electrical Engineering from Universiti Teknologi Malaysia (UTM) in 2014. She spent 3 months at University of Birmingham, U.K., as a visiting Ph.D. student. She had also previously served as a senior assistant researcher at TM Research \& Development, Malaysia, for 3 years. She is currently a senior lecturer at Advanced Informatics School, Universiti Teknologi Malaysia. Her research interests include antennas, artificial magnetic conductor, metamaterials and body-area wireless communications.

Mohamad Kamal A. RAHIM was born in Alor Setar, Kedah, Malaysia in 1964. He obtained his B.Eng. in Electrical and Electronic from University of Strathclyde, U.K. in 1987 and M.Eng. from University of New South Wales, Australia in 1992. He received his Ph.D. in the field of Wideband Active Antenna. He is a Professor at Communications Engineering Department, Faculty of Electrical Enginnering at Universiti Teknologi Malaysia. Professor Mohamad Kamal is a senior member of IEEE since 2007. His research interest includes antennas, metamaterials, body-area communications and reconfigurable antennas.

Peter S. HALL is a Professor of Communications Engineering at School of Electronic, Electrical and Computer Engineering, University of Birmingham, U.K. Professor Hall is a Fellow of the IEE and the IEEE and a past IEEE Distinguished Lecturer. He is also a past Chairman of the IEE Antennas and Propagation Professional Group. He has researched extensively in the areas of antennas, propagation and antenna measurements. He has published 5 books, over 350 learned papers and taken various patents. These publications have earned many awards, including the 1990 IEE Rayleigh Book Award for the Handbook of Microstrip Antennas.

Noor Asmawati SAMSURI was born in 1980. She obtained her B.Eng. in Electrical (Telecommunication) from Universiti Teknologi Malaysia. She received both her M.Sc. and Ph.D. from Loughborough University, U. K. in 2004 and 2009 respectively. She is currently a senior lecturer at the Faculty of Electrical Engineering, Universiti Teknologi Malaysia. Her research specialization includes antennas, antenna interaction and coupling with human and metallic jewelry and EM field absorption (SAR).

Mohd Ezwan JALIL was born in Johor, Malaysia in 1989. He received his B.Eng. in Electrical (Telecommunication) from Universiti Teknologi Malaysia. He completed his M.Eng. in 2013, also from Universiti Teknologi Malaysia. He is currently pursuing Ph.D. in the field of antenna and RF devices at Universiti Teknologi Malaysia. His research area includes chipless RFID tag, textile antennas and body-area communications.

Mohd Fareq Abd. MALEK is an Associate Professor at Universiti Malaysia Perlis (UMP). He is currently the Dean of School of Electrical System at UMP. He obtained his B.Eng. in Electronic and Communication from University of Birmingham. He then received his M.Sc. in Microelectronic Systems and Telecommunications and Ph.D. in Radio Frequency, Microwave and Free Electron Maser; both from University of Liverpool. His research interest includes radio frequency, microwave, waveguide, free electron maser, travelling wave tube, antennas, broadband wireless. 